\title{
Diffusion Tensor Imaging of the Spinal Canal in Quantitative Assessment of Patients with Lumbar Spinal Canal Stenosis
}

\author{
Masaki Norimoto ${ }^{1}$, Yawara Eguchi ${ }^{2}$, Hirohito Kanamoto ${ }^{3}$, Yasuhiro Oikawa ${ }^{4}$, Koji Matsumoto ${ }^{5}$, \\ Yoshitada Masuda ${ }^{5}$, Takeo Furuya ${ }^{1}$, Sumihisa Orita ${ }^{1}$, Kazuhide Inage ${ }^{1}$, Satoshi Maki ${ }^{1}$, Yasuhiro Shiga ${ }^{1}$, \\ Hideyuki Kinoshita ${ }^{6}$, Koki Abe ${ }^{1}$, Masahiro Inoue ${ }^{1}$, Tomotaka Umimura ${ }^{1}$, Takashi Sato ${ }^{1}$, \\ Masashi Sato ${ }^{1}$, Masahiro Suzuki ${ }^{1}$, Keigo Enomoto ${ }^{1}$, Seiji Ohtori ${ }^{1}$ \\ ${ }^{I}$ Department of Orthopaedic Surgery, Graduate School of Medicine, Chiba University, Chiba, Japan \\ ${ }^{2}$ Department of Orthopaedic Surgery, Shimoshizu National Hospital, Yotsukaido, Japan \\ ${ }^{3}$ Kanamoto Orthopaedic Clinic, Numazu, Japan \\ ${ }^{4}$ Department of Orthopaedic Surgery, Chiba Children's Hospital, Chiba, Japan \\ ${ }^{5}$ Department of Radiology, Chiba University Hospital, Chiba, Japan \\ ${ }^{6}$ Department of Orthopaedic Surgery, Chiba Cancer Center, Chiba, Japan
}

\section{Study Design: Retrospective observational study.}

Purpose: Lumbar spinal stenosis (LSS) has traditionally been evaluated morphologically, there is a paucity of literature on quantitative assessment of LSS. The purpose of this study was to investigate whether intraspinal diffusion tensor imaging (DTI) parameters such as apparent diffusion coefficient (ADC) and fractional anisotropy (FA) are useful for assessing LSS.

Overview of Literature: Quantitative assessment of LSS is challenging.

Methods: Study participants comprised five healthy volunteers (mean age, 27.2 years) and 27 patients with LSS (mean age, 58.4 years) who were individually assessed using 3.0 Tesla magnetic resonance imaging. Intraspinal ADC and FA values of 10 intervertebral discs from healthy volunteers and 52 intervertebral discs from LSS patients were measured. Also, intraspinal canal area, Schizas classification (A: normal, B: mild stenosis, C: severe stenosis) and correlations with symptoms were investigated. Clinical symptoms were checked for the presence of low back pain (LBP), intermittent claudication (IMC), and bladder and bowel dysfunction (BBD).

Results: Compared to healthy individuals, LSS patients had significantly lower ADC $(p<0.05)$ and significantly higher FA values $(p<0.01)$. In Schizas classification, stenosis worsened from A to $C$. ADC values decreased significantly while FA values increased significantly in that order $(p<0.05)$. A positive correlation was found between intraspinal canal area and $A D C$ values $(r=0.63, p<0.01)$ and a negative correlation between intraspinal canal area and FA values $(p=-0.61, p<0.01)$. No correlations were noted between LBP and ADC or FA values. On the other hand, $A D C$ values were significantly lower $(p<0.05)$ and $F A$ values were significantly higher $(p<0.05)$ in patients with IMC or BBD.

Conclusions: Intraspinal DTI parameters such as ADC and FA values were associated with the Schizas classification, intraspinal canal area, and clinical symptoms, suggesting that ADC and FA may be useful for quantitative assessment of LSS.

Keywords: Diffusion tensor imaging; Apparent diffusion coefficient; Fractional anisotropy; Lumbar spinal stenosis

Received Jan 20, 2020; Revised Feb 21, 2020; Accepted Mar 10, 2020

Corresponding author: Masaki Norimoto

Department of Orthopaedic Surgery, Graduate School of Medicine, Chiba University, 1-8-1 Inohana, Chuo-ku, Chiba, 260-8670, Japan Tel: +81-43-226-2117, Fax: +81-43-226-2116, E-mail: seikei@office.chiba-u.jp 


\section{Introduction}

In recent years, the incidence of lumbar spinal stenosis (LSS) has been growing with an increase in the aging population. In a large-scale study of 938 patients with a mean age of 66 years, its prevalence was approximately $10 \%$. LSS impairs patients' quality of life and has now become a major social issue [1]. In some cases of LSS, the visualized site of maximum stenosis does not reveal the site of neurological impairment, which may lead to difficulties in determining surgical indications [2].

Schizas et al. [3] performed morphological evaluations based on changes in intraspinal cauda equina innervation in spinal canal stenosis. However, intraspinal lesions were primarily diagnosed based on morphology alone, and quantitative assessment methods have been unavailable.

Magnetic resonance imaging (MRI) allows acquisition of diffusion-weighted image (DWI) by enhancing water molecule movements (diffusion) with the application of motion probing gradient from one direction to proton diffusion movement [4-7]. DWIs are widely used to diagnose acute cerebral infarctions [8] or other central neurological deseases $[9,10]$. Nerve fibers are anisotropic tissue as they have axon cellular membrane and myelin sheaths, which interrupt the water molecule diffusion. The selective recording of this data is called diffusion tensor imaging (DTI), and the visualization of nerve fibers via DTI is known as tractography. The parameter representing anisotropy includes fractional anisotropy (FA) which ranges from 0 to 1 . FA has a of 1 in anisotropic tissues and approaches the value of 0 in water. Some studies reported the usefulness of DTI in evaluating demyelinating diseases (such as multiple sclerosis) or chronic peripheral nerve compression lesions (such as carpal tunnel syndrome) $[11,12]$. In intact myelinated nerves, tissue myelin sheath restricts water molecule diffusion, thereby results in potent anisotropy. However, in demyelinated conditions such as nerve injury, there is a reduction in tissue anisotropy which causes a decrease in FA value $[13,14]$. In terms of the lumbar spinal region, there are studies reported on the use of DTI to evaluate the lumbar nerve root [15-20]. However, imaging of the spinal cord is challenging due to technical limitations such as the relatively small size of the spinal cord, susceptibility artifacts from tissue-bone interfaces and the motion artifacts arising from respiratory activity. We previously reported that in patients with lumbar foraminal stenosis, the tract was blocked at the stenotic site and apparent diffusion coefficient (ADC) values and FA values were correlated with clinical symptoms $[15,16]$. Therefore, DTI parameters can predict neurological severity and serves potential tools for functional diagnosis of LSS. Despite advances in DTI of lumbar nerve, intraspinal nerves are affected by the magnetic susceptibility anisotropy of cerebrospinal fluid (CSF), hence making DTI assessment difficult.

Kanamoto et al. [21] found that reduced field of view (rFOV) with localized excitation (focus) can be used to acquire a high resolution and high intraspinal signal-tonoise image, which overcome the limitations associated with conventional methods.

Our purpose was to investigate the correlation between clinical symptoms and morphological findings of spinal canals using the DTI parameters ADC and FA, and to determine their usefulness for quantitative assessment of LSS.

\section{Materials and Methods}

\section{Participants}

All procedures in this study were performed in accordance with the ethical standards of the institutional and/ or national research committee and the 1964 Helsinki declaration and its later amendments or comparable ethical standards. Informed consent was obtained from all participants prior to participation in any study-related procedures. We performed 3.0 Tesla MRI in five healthy volunteers (four men, one woman; mean age, 27.2 years) and 27 patients with LSS (14 men, 13 women; mean age, 58.4 years). In the healthy volunteers, two discs between L4/5 and L5/S1 were imaged, resulted in a total of 10 intervertebral discs. On the other hand, MRI scan of one to three consecutive intervertebral spaces in LSS patients was taken depending on the stenotic site. A total of 52 intervertebral discs were examined: (1) one disc: L4/5 in one patient and L5/S1 in three patients; (2) two discs: L2/3 and $\mathrm{L} 3 / 4$ in one patient, $\mathrm{L} 3 / 4$, and $\mathrm{L} 4 / 5$ in five patients, L4/5 and L5/S1 in 15 patients; and (3) three discs L3/4, L4/5, L5/S in two patients.

LSS was diagnosed based on spinal myelogram, MRI, and computed tomography findings after spinal myelogram by three spinal surgery specialists. Exclusion criteria included history of lumbar spine surgery, vertebral osteomyelitis, spinal tumors, or spinal trauma. Each intervertebral disc was classified based on a T2-weighted axial image 
according to the Schizas classification as A (A1-A4), B, C, or D (Fig. 1). No patients in our study had lesions in class D.

\section{Conventional magnetic resonance imaging}

All participants were imaged using a 3.0 Tesla MRI (Discovery MR 750; GE Healthcare, Waukesha, WI, USA), which provided sagittal T1-weighted (T1W) and T2weighted $(\mathrm{T} 2 \mathrm{~W})$ images and axial T2W images. For the sagittal imaging, the acquisition settings were: field of view (FOV), $260 \times 260 \mathrm{~mm}$; slice thickness, $3 \mathrm{~mm}$; and echo time (TE)/time of repetition (TR), 8.4/530 ms (T1W) or 104/4,540 ms (T2W). For the axial imaging, the acquisition settings were: FOV, $160 \times 160 \mathrm{~mm}$; slice thickness, 4 $\mathrm{mm}$; and TE/TR, 100/4,500 ms.

\section{Diffusion tensor imaging protocol}

The DTI protocol included DTI sequences in the axial plane with the rFOV [20]. The participants were scanned in a supine position using Sense XL Torso coil (Philips Healthcare, Best, The Netherlands). The rFOV scan was performed using a special sensitivity array encoding technique (factor: 2, spectral-spatial radio frequency pulse and water excitation method), a diffusion-weighted spinecho single-shot echo-planar imaging with free breathing technique, 11 different diffusion gradients, and a $b$-value of $800 \mathrm{sec} / \mathrm{mm}^{2}$. The rFOV was created using FOV optimized and constrained undistorted single-shot sequence sequence and two-dimensional spatially selective echoplanar radiofrequency excitation pulse (GE Healthcare). The 16 slices for rFOV (contiguous thickness, $3 \mathrm{~mm}$ ) were acquired from L3 to S1 nerve roots. The parameters were

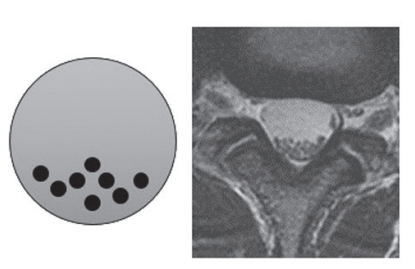

Class A1

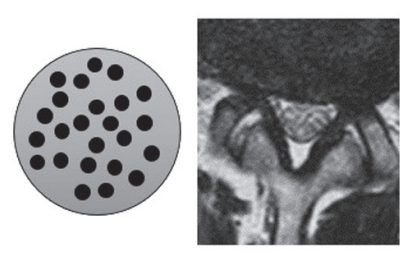

Classs B

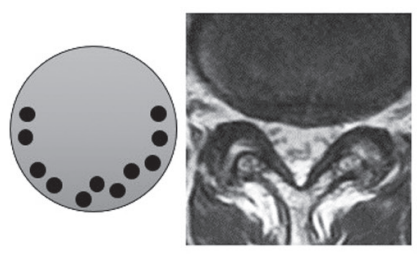

Class A2

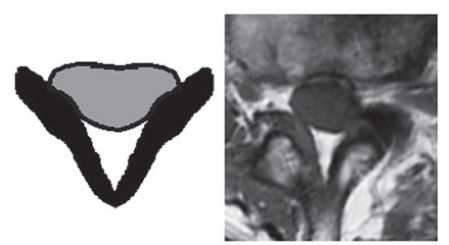

Class C

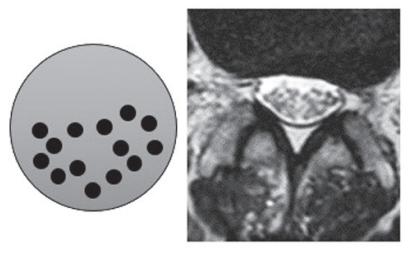

Class A3

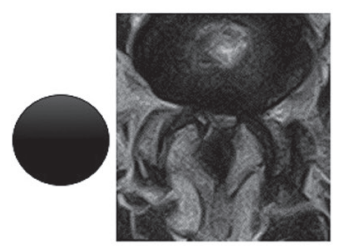

Class D

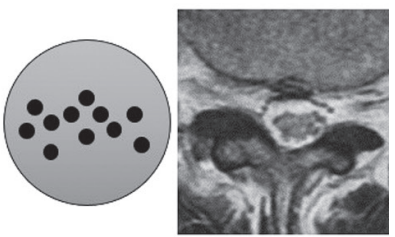

Class A4

Fig. 1. Schizas classification. Based on the innervation morphology of cauda equina, disease is classified into class $\mathrm{A}$ to D. Class A: normal (1-4); class B: mild stenosis; class C: moderate to severe stenosis; and class D: severe stenosis.
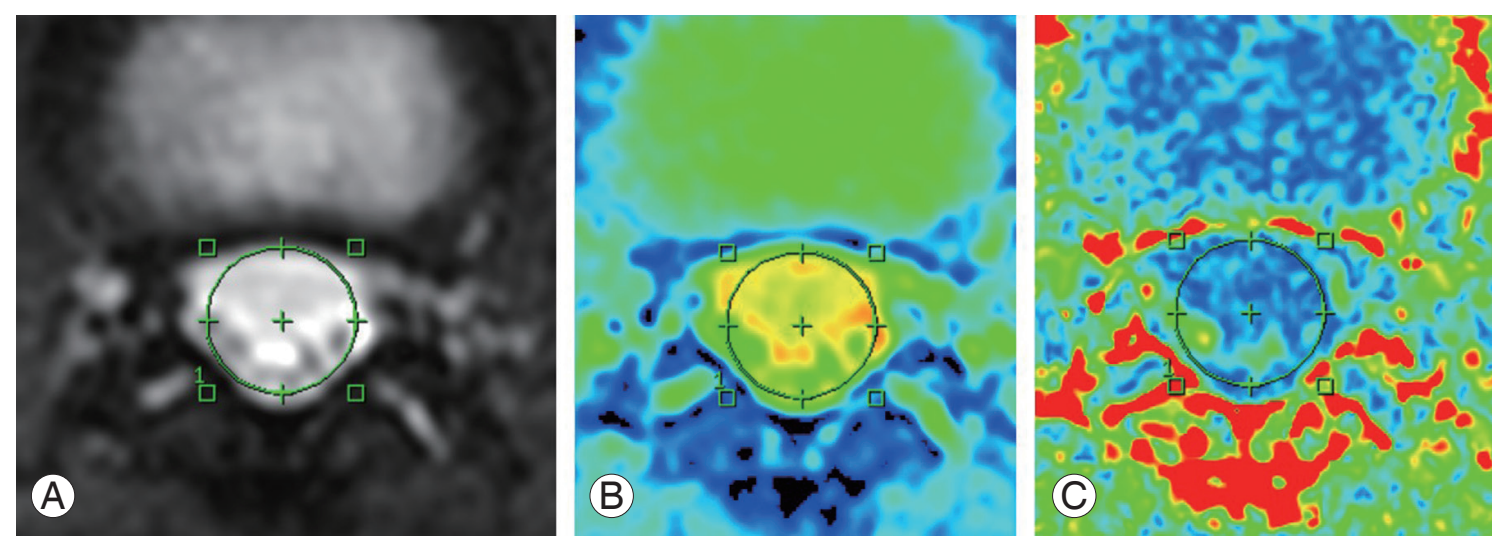

Fig. 2. (A-C) Setting of ROI. Intraspinal ROI was established on an axial image at intervertebral disc levels and intraspinal canal area, ADC, and FA were automatically measured. The red area indicates high signal intensity and blue area indicates low signal intensity. ROI, regions of interest; ADC, apparent diffusion coefficient; FA, fractional anisotropy. 
as follows: TR, 6,000 ms; TE, $55.6 \mathrm{~ms} ; 6$ excitations, FOV, $100 \times 60 \mathrm{~mm}$; matrix, $68 \times 34$; voxel size, $1.47 \times 1.76 \times 3 \mathrm{~mm}$; and total acquisition time, 7 minutes 18 seconds.

\section{Image analysis}

Axial images were obtained at each intervertebral level and an intraspinal region of interest was established. ADC, FA and intraspinal canal areas were each measured twice (Fig. 2). Two spinal surgery specialists (M.N. and T.S.) with 10 or more years of experience took these measurements. Intra-observer reliability (M.N.) and interobserver reliability (M.N. versus T.S.) were also calculated.

Clinical symptoms included low back pain (LBP). A visual analysis scale score of 5 or greater (0 [no pain] to 10 [greatest pain imaginable]) was defined positive for LBP. Intermittent claudication (IMC) was diagnosed if lower limb pain forced the patient to stop after walking for $500 \mathrm{~m}$ or less and hence defined as positive for IMC. The Japanese Orthopedic Association (JOA; 0-29 points) scoring system was used to assess bladder rectal disorder (bladder and bowel dysfunction [BBD]). The normal JOA score is 29 points, based on three subjective symptoms ( 9 points), three clinical signs including straight-leg raising (6 points), seven activities of daily living (14 points), and bladder disorder ( -6 points). Bladder function scores for mild dysfunction: frequency, residual urine ( -3 points), and severe dysfunction: urinary retention, incontinence ( -6 points) were defined as positive for BBD.

$\mathrm{ADC}$ and FA values were assessed in LSS patients at the site of most severe stenosis as follows: (1) ADC and FA values, and intraspinal canal areas in healthy subjects were compared to LSS patients. (2) ADC and FA values were compared to Schizas classification and intraspinal canal areas in LSS patients. (3) ADC and FA values, and clinical symptoms (LBP, IMC, and BBD) were studied in LSS patients.

\section{Statistical analysis}

Statistical analyses were performed using StatView software ver. 5.0 (Stata Corp., College Station, TX, USA). For each parameter, differences between both groups or clinical severities were evaluated using unpaired $t$-test. Pearson correlation coefficients were calculated to determine the correlation between diameter of spinal canal and DTI parameters in the LSS group. All data were expressed as

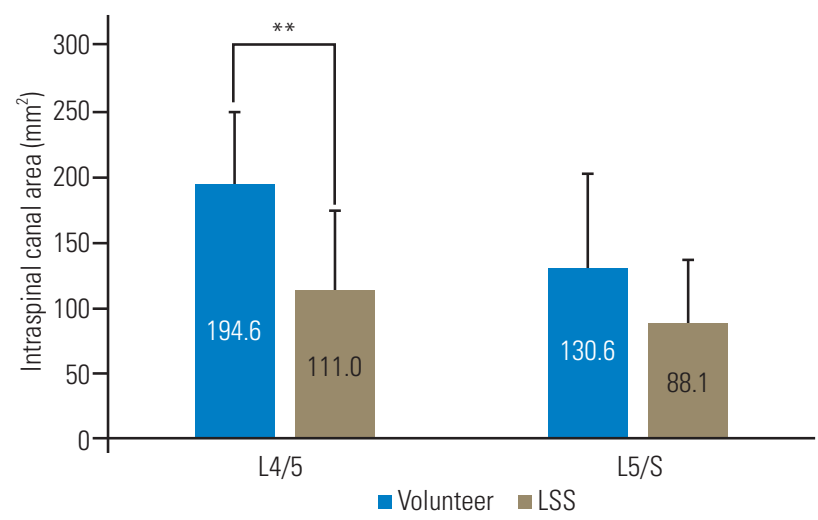

Fig. 3. Comparison of intraspinal canal areas in healthy subjects and LSS patients. At the $L 4 / 5$ level, healthy individuals had significantly larger intraspinal canal area than LSS patients. Conversely, at the L5/S level, there were no significant difference between patients and healthy subjects in intraspinal canal area. LSS, lumbar spinal stenosis. ${ }^{* *} p<0.01$.

mean \pm standard deviation. A threshold probability value of $p<0.05$ was considered statistically significant.

\section{Results}

\section{Comparison of ADC and FA values, and intraspinal canal areas in healthy subjects and LSS patients}

Intraspinal canal areas in healthy subjects were 194.6 \pm 55.6 $\mathrm{mm}^{2}$ at $\mathrm{L} 4$ and $130.6 \pm 71.9 \mathrm{~mm}^{2}$ at L5/S. In LSS patients, the intraspinal canal area was $111.0 \pm 64.4 \mathrm{~mm}^{2}$ at $\mathrm{L} 4 / 5$ and $88.1 \pm 48.7 \mathrm{~mm}^{2}$ at L5/S. Areas were significantly smaller at the L4/5 in the LLS patient group. No statistically significant differences were noted at L5/S (Fig. 3).

ADC values of normal healthy individuals at $\mathrm{L} 4 / 5$ were $2.72 \pm 0.18 \times 10^{-3} \mathrm{~mm}^{2} / \mathrm{sec}$ and $2.77 \pm 0.18 \times 10^{-3} \mathrm{~mm}^{2} /$ $\mathrm{sec}$ at $\mathrm{L} 5 / \mathrm{S}$. The ADC values of LLS patient values were significantly lower at both $\mathrm{L} 4 / 5$ and $\mathrm{L} 5 / \mathrm{S}$, as evidenced by ADC values of $2.24 \pm 0.68 \times 10^{-3} \mathrm{~mm}^{2} / \mathrm{sec}$ at $\mathrm{L} 4 / 5$ and $2.50 \pm 0.49 \times 10^{-3} \mathrm{~mm}^{2} / \mathrm{sec}$ at L5/S (Fig. $4 \mathrm{~A}$ ).

In healthy subjects, FA values were $0.121 \pm 0.017$ at $\mathrm{L} 4 / 5$ and $0.115 \pm 0.024$ at L5/S. Meanwhile, FA values in LLS patients were $0.225 \pm 0.119$ at $\mathrm{L} 4 / 5$ and $0.156 \pm 0.058$ at $\mathrm{L} 5 / \mathrm{S}$, indicating that the FA values were significantly higher at both L4/5 and L5/S sites in the LSS patients (Fig. 4B).

\section{ADC and FA values compared to Schizas classifica- tion and intraspinal canal area in LSS patients}

ADCs were $1.44 \pm 0.27 \times 10^{-3} \mathrm{~mm}^{2} / \mathrm{sec}$ in Schizas class C, $1.89 \pm 0.35 \times 10^{-3} \mathrm{~mm}^{2} / \mathrm{sec}$ in class B and $2.63 \pm 0.41 \times 10^{-3}$ 

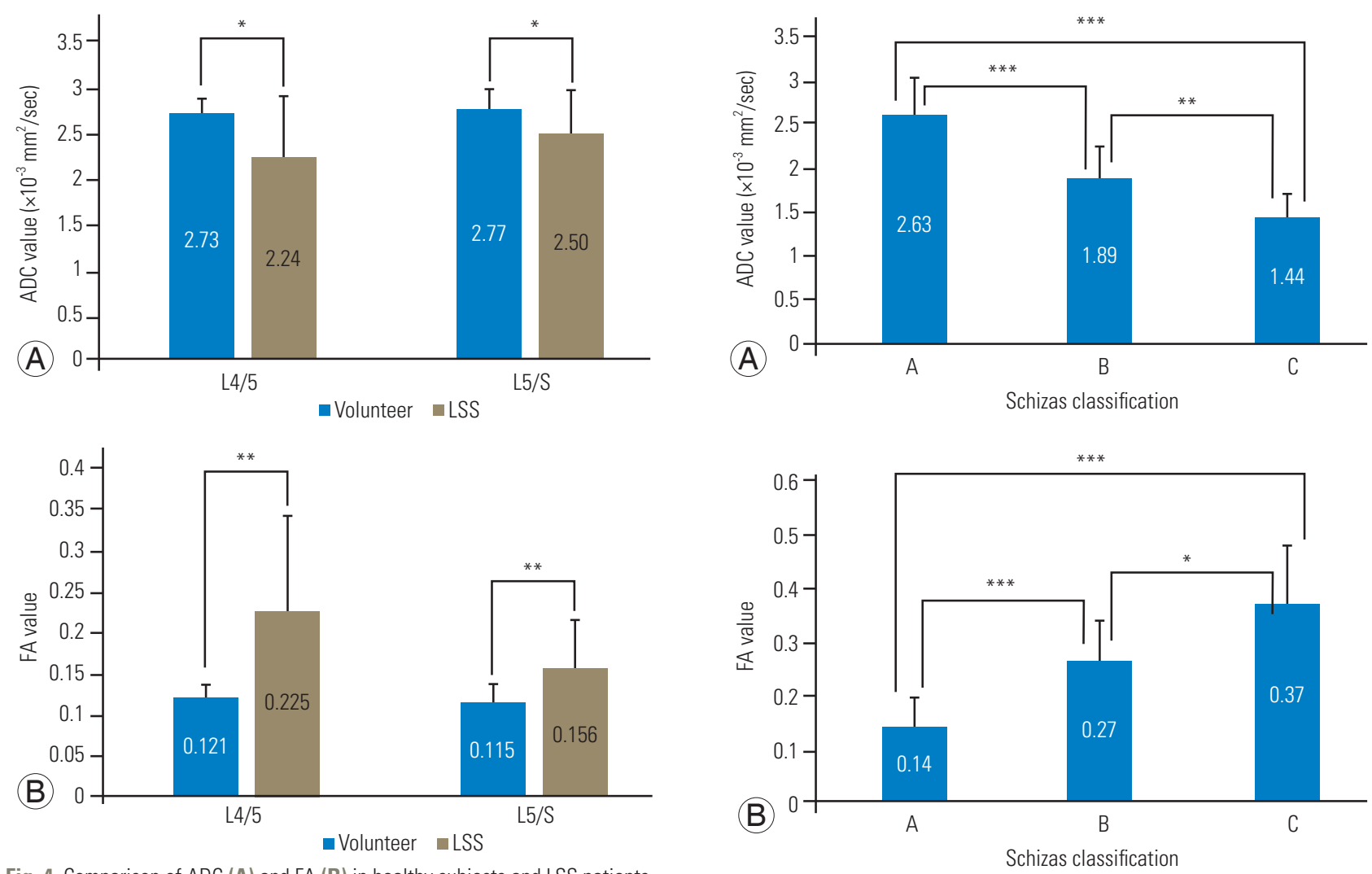

Fig. 4. Comparison of $A D C(A)$ and $F A(B)$ in healthy subjects and LSS patients. (A) ADC was significantly higher in healthy subjects at both the $L 4 / 5$ and $L 5 / S$ levels. (B) FA was significantly lower in healthy subjects at both L4/5 and L5/ $S$ levels. ADC, apparent diffusion coefficient; FA, fractional anisotropy; LSS, lumbar spinal stenosis. ${ }^{*} p<0.05{ }^{* *} p<0.01$.

$\mathrm{mm}^{2} / \mathrm{sec}$ in class $\mathrm{A}$, showing that $\mathrm{ADC}$ values decreased significantly from class A to B to C (Fig. 5A). Conversely, FA values significantly increased with disease aggravation where class $C$ was $0.372 \pm 0.105$, class B was $0.267 \pm 0.074$ and class A was $0.144 \pm 0.055$, class $C$ had the highest FA value, followed by class B and A (Fig. 5B).

Comparisons between ADC and intraspinal canal area revealed a statistically significant positive correlation at all levels with an overall correlation coefficient of 0.62 , where correlation coefficient was 0.81 at $\mathrm{L} 3 / 4,0.72$ at $\mathrm{L} 4 /$, and 0.50 at $\mathrm{L} 5 / \mathrm{S}$. The correlation at L5/S was relatively weaker at other levels (Fig. 6A).

The negative correlation between FA and intraspinal canal area was statistically significant at all levels with a correlation coefficient of -0.61 . At L3/4, L4/5, and L5/S, its correlation coefficient was $-0.83,-0.75$, and -0.49 , respectively. As for ADC, despite significant correlations at all levels, the association was slightly weaker at L5/S (Fig. 6B).

\section{ADC and FA values, and clinical symptoms (LBP, IMC, and BBD) in LSS patients}

LLS patients with LBP had mean ADC value of $2.21 \pm 0.64 \times 10^{-3}$ $\mathrm{mm}^{2} / \mathrm{sec}$ and FA value of $0.227 \pm 0.127$. On the other side, LLS patients without LBP had mean ADC value of $2.09 \pm 0.73 \times 10^{-3} \mathrm{~mm}^{2} / \mathrm{sec}$ and FA value of $0.252 \pm 0.133$. However, there were no statistically significant differences in ADC or FA between LLS patients with or without LBP (Fig. 7A).

LLS patients with IMC had an ADC of $1.83 \pm 0.66 \times 10^{-3}$ $\mathrm{mm}^{2} / \mathrm{sec}$ and FA of $0.304 \pm 0.137$. Those patients without IMC had an ADC of $2.50 \pm 0.67 \times 10^{-3} \mathrm{~mm}^{2} / \mathrm{sec}$ and FA of $0.180 \pm 0.088$. Patients with IMC had a significantly lower $\mathrm{ADC}$ and significantly higher FA (Fig. 7B).

Patients with BBD had an ADC of $1.26 \pm 0.34 \times 10^{-3} \mathrm{~mm}^{2} /$ sec and FA of $0.369 \pm 0.085$. Those without BBD had an ADC of $2.29 \pm 0.69 \times 10^{-3} \mathrm{~mm}^{2} / \mathrm{sec}$ and FA of $0.223 \pm 0.125$. LLS patients with $\mathrm{BBD}$ demonstrated significantly lower ADCs and significantly higher FA values (Fig. 7C). 

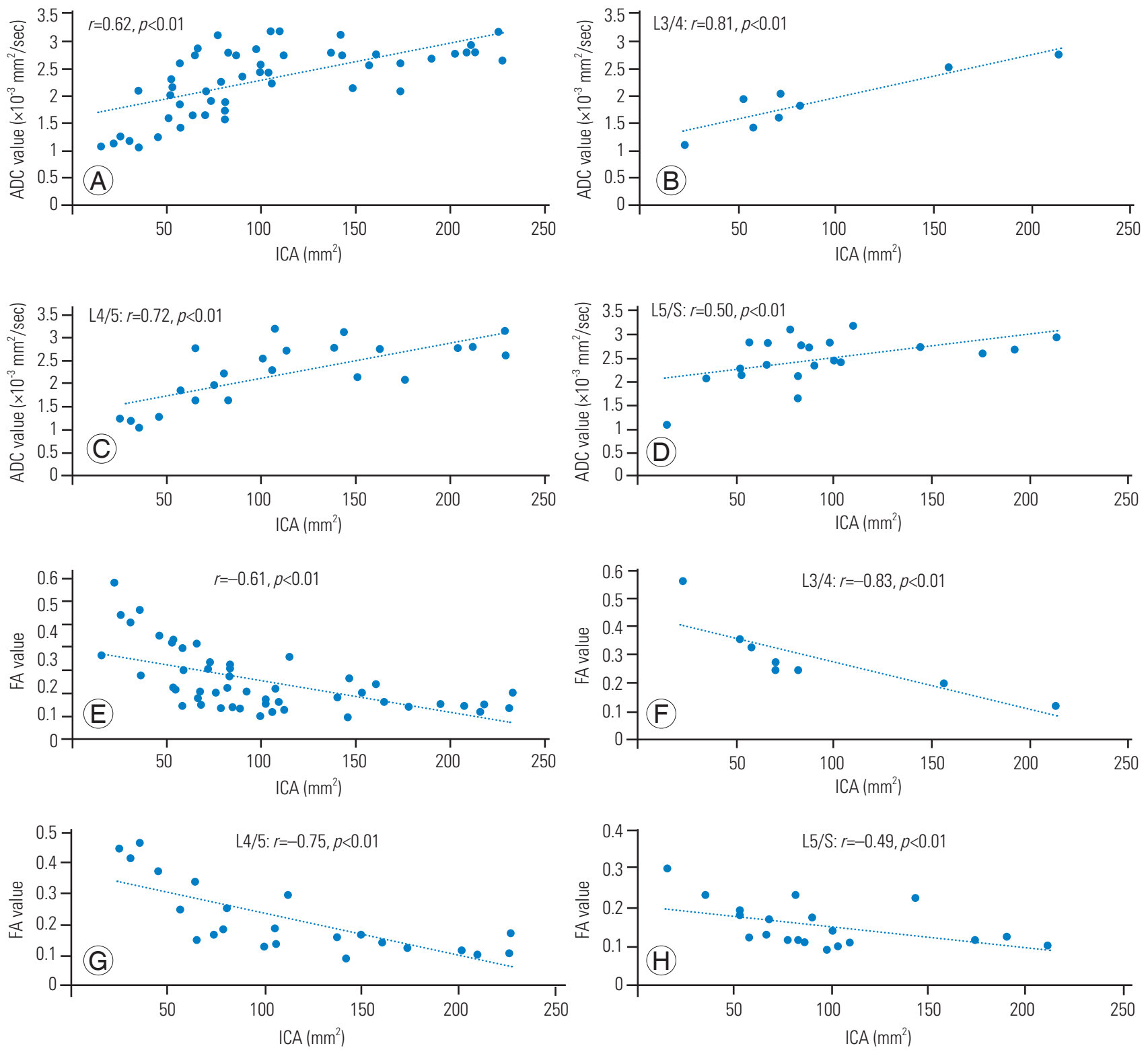

Fig. 6. Correlation between ADC (A-D) and FA (E-H) with ICA. (A-D) ADC values and ICA showed a significant positive correlation. Correlation was slightly weaker at the L5/S level compared to other intervertebral levels. (E-H) FA values and ICA showed a significant negative correlation. Correlation was slightly weaker at the L5/ S level compared to other levels. ADC, apparent diffusion coefficient; FA, fractional anisotropy; ICA, intraspinal canal area.

Intra-individual reliability of observers for ADC values was $r=0.98$, and interindividual reliability was $r=0.97$. For the FA values, intra-individual reliability was $r=0.98$ while interindividual reliability was $r=0.97$.

\section{Discussion}

In this study, DTI parameters ADC and FA were investigated for the correlations of spinal canal morphology and clinical symptoms. Compared to healthy individuals, LSS patients have significantly lower ADC values and significantly higher FA values. According to the Schizas classification, A has the least stenosis, B has more, and C has the most stenosis. ADC decreased significantly from class A to $B$ to $C$, while the FA value increased significantly in the same order. Intraspinal canal area showed a positive correlation with ADC values while it had a negative correlation with FA values. No correlation was noted to LBP, but patients with IMC and BBD had significantly lower $\mathrm{ADC}$ and significantly higher FA values. Intraspinal ADC 

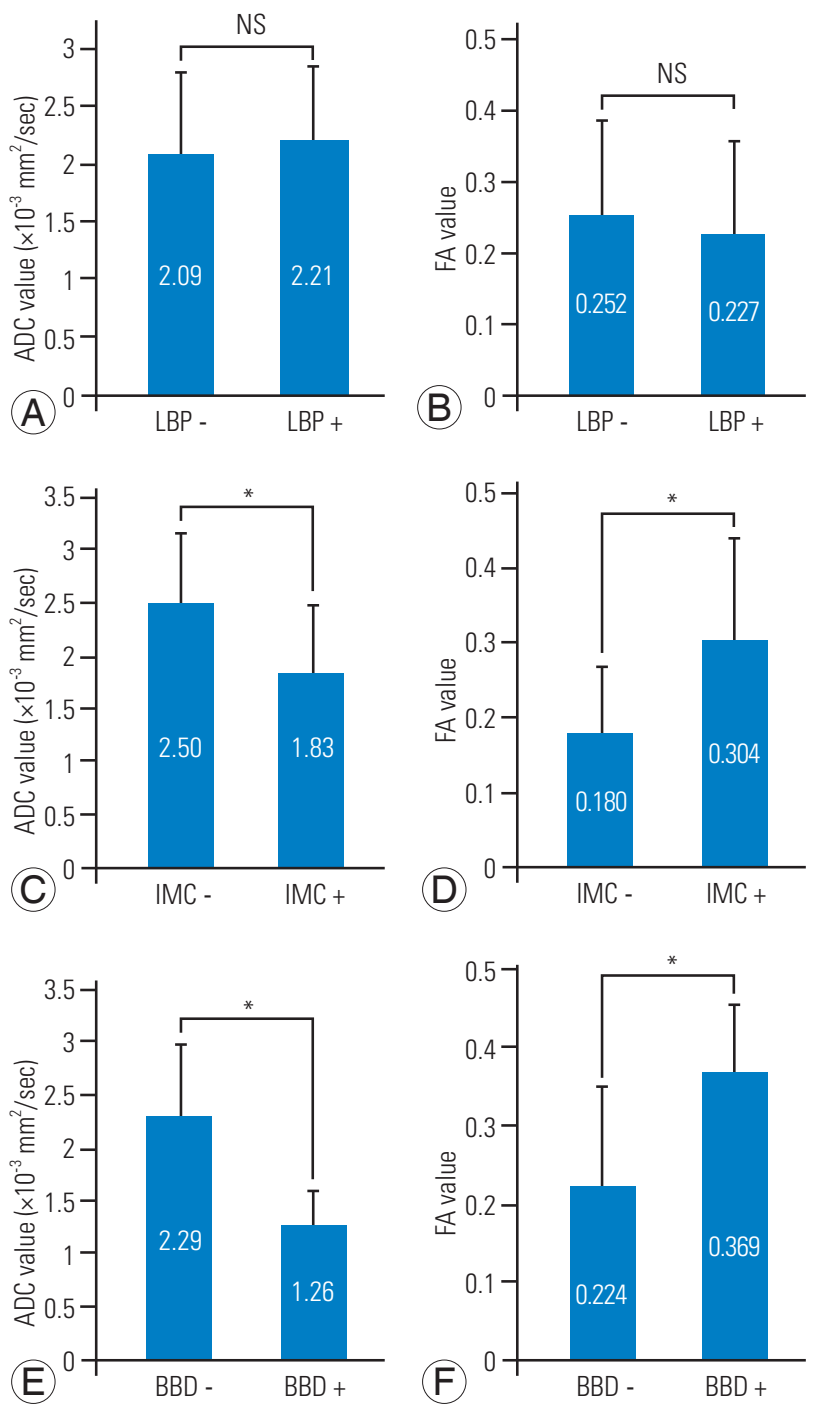

Fig. 7. Association between $A D C$ and FA with clinical symptoms. (A, B) Association with LBP: ADC and FA were compared in the groups with and without LBP. No significant differences in ADC and FA were noted in both groups. (C, D) Association with IMC: ADC and FA values were compared in the groups with and without IMC. ADC was significantly lower and FA significantly higher in the groups with IMC. (E, F) Association with BBD: ADC and FA values were compared in the groups with and without BBD. ADC was significantly lower and FA significantly higher in the groups with $\mathrm{BBD}$. ADC, apparent diffusion coefficient; FA, fractional anisotropy; LBP, low back pain; IMC, intermittent claudication; BBD, bladder and bowel dysfunction; NS, not significant. $p<0.05$.

and FA were correlated with the Schizas classification, intraspinal canal area, and clinical symptoms, indicating that intraspinal ADC and FA may be useful for the quantitative assessment of LSS.

Recent reports have shown that sedimentation signs are important prognosis indicators in spinal canal stenosis. In a large-scale prospective study called SPORT (Spine Patient Outcomes Research Trial), 66\% of spinal canal ste- nosis cases showed a positive sedimentation sign wherein the positive sedimentation sign group was often resistant to conservative therapy and hence transitioning to surgery indication [22].

In conventional MRI, false positive findings may present in asymptomatic individuals, thus making it extremely difficult to predict postoperative prognosis or nerve damage. With regard to IMC, Hamanishi et al. [23] demonstrated that developmental narrowing of the spinal canal was found not only in the IMC group, but also in the radicular pain group. Regarding neuropathic bladder, Inui et al. [24] presented that there was no significant difference in the cross-sectional area of dural sac between the patients with positive and negative neuropathic bladder, and dural sac anteroposterior diameter might be an important predictive factor of neuropathic bladder. DTI is a noninvasive way to effectively trace the nerve fiber bundle and quantitatively evaluate the nerve injury. Recently, increasing attention has been directed to the application of DTI and tractography on the lumbar nerve root [15-20].

Generally, increased ADC may be caused by inflammation or edema, whereas decreased FA may reflect damaged tissue microstructure, demyelination, axonal loss, or increased isotropic water volume. This study showed that ADC values were significantly lower in LSS patients compared to healthy individuals, while FA values were significantly higher, these findings are in contrary to previous findings on peripheral nerve DTI parameters.

In normal physiological condition, CSF accounts for a large proportion of dura sacs. A high diffusion of water molecules causes higher ADC values, and since the water molecules show isotropic diffusion, FA is believed to decrease. In intervertebral discs with stenosis, CSF accounts for a smaller percentage, causing cauda equina density to increase while water molecules disperse along the nerve fibers. In other words, at the spinal canal stenotic sites, water molecule diffusion is restricted, thereby causing a decrease in ADC values with increased anisotropic dispersion, resulting in increased FA values. These results reflect the diffusion and anisotropy of CSF from the nerve per se. Although ADC and FA values did not correlate with LBP, these parameters correlate with severe disease such as IMC and BBD, and may serve to quantify the neurological symptoms associated with LSS. The average $\mathrm{ADC}$ value for patients presenting with $\mathrm{BBD}$ was $1.26 \times 10^{-3}$ and the average FA value was 0.369 . An ADC value of less than $1.26 \times 10^{-3} \mathrm{~mm}^{2} / \mathrm{sec}$ and $\mathrm{FA}$ value of 0.369 or more at 
the responsible intervertebral level in LSS patients may be considered a standard for surgical indication.

Our study has several limitations. (1) Only a small number of subjects were investigated, hence confirmation of our findings requires a larger population. (2) No functional assessments of LBP such as Roland-Morris disability questionnaire or Oswestry disability index were performed. (3) The healthy individuals (mean age, 27 years) were significantly younger than the LSS patients (mean age, 58 years). (4) Patients were not evaluated postoperatively. (5) Regarding the clinical symptoms, there was a significant difference between FA and ADC values when divided by the presence or absence of IMC or BBD, but the correlation with the severity of clinical symptoms was not examined. Therefore, further investigations will be required.

\section{Conclusions}

Correlations between DTI parameters (ADC and FA values), morphological evaluation of the spinal column and clinical symptoms were determined in the LSS patients. Thus far, assessment of LSS has depended on morphology and there has been little literature on the quantitative evaluation methods. Our findings show that LSS patients have significantly lower ADC values and significantly higher FA values compared to healthy persons. When ADC and FA values were evaluated in association with Schizas class and the effects on intraspinal canal area were compared, ADC values showed a positive correlation, while FA values showed a negative correlation to the area. No correlation to clinical symptoms of LBP was apparent, while patients with IMC or BBD had significantly lower ADC and significantly higher FA values. Associations were identified between intraspinal ADC and FA values and Schizas classification, intraspinal canal area, and clinical symptoms, indicating that these ADC and FA may be useful to assess LSS quantitatively.

\section{Conflict of Interest}

No potential conflict of interest relevant to this article was reported.

\section{References}

1. Ishimoto Y, Yoshimura N, Muraki S, et al. Associa- tions between radiographic lumbar spinal stenosis and clinical symptoms in the general population: the Wakayama Spine Study. Osteoarthritis Cartilage 2013;21:783-8.

2. Eguchi Y, Ohtori S, Toyone T, Ozawa T, Yamauchi K, Yamashita M. Surgical experience in cases of L5 and S1 symptoms caused by upper lumbar spinal stenosis of L2-L3 and L3-L4. J Spine 2012;1:1000105.

3. Schizas C, Theumann N, Burn A, et al. Qualitative grading of severity of lumbar spinal stenosis based on the morphology of the dural sac on magnetic resonance images. Spine (Phila Pa 1976) 2010;35:191924.

4. Basser PJ, Jones DK. Diffusion-tensor MRI: theory, experimental design and data analysis: a technical review. NMR Biomed 2002;15:456-67.

5. Beaulieu C, Allen PS. Determinants of anisotropic water diffusion in nerves. Magn Reson Med 1994;31:394-400.

6. Beaulieu C, Does MD, Snyder RE, Allen PS. Changes in water diffusion due to Wallerian degeneration in peripheral nerve. Magn Reson Med 1996;36:627-31.

7. Basser PJ, Pierpaoli C. Microstructural and physiological features of tissues elucidated by quantitativediffusion-tensor MRI. J Magn Reson B 1996;111:20919.

8. Minematsu K, Li L, Fisher M, Sotak CH, Davis MA, Fiandaca MS. Diffusion-weighted magnetic resonance imaging: rapid and quantitative detection of focal brain ischemia. Neurology 1992;42:235-40.

9. Lin X, Tench CR, Morgan PS, Constantinescu CS. Use of combined conventional and quantitative MRI to quantify pathology related to cognitive impairment in multiple sclerosis. J Neurol Neurosurg Psychiatry 2008;79:437-41.

10. Mamata H, Jolesz FA, Maier SE. Apparent diffusion coefficient and fractional anisotropy in spinal cord: age and cervical spondylosis-related changes. J Magn Reson Imaging 2005;22:38-43.

11. Takagi T, Nakamura M, Yamada M, et al. Visualization of peripheral nerve degeneration and regeneration: monitoring with diffusion tensor tractography. Neuroimage 2009;44:884-92.

12. Mac Donald CL, Dikranian K, Bayly P, Holtzman D, Brody D. Diffusion tensor imaging reliably detects experimental traumatic axonal injury and indicates approximate time of injury: version 2. J Neurosci 
2007;27:11869-76.

13. Khalil C, Hancart C, Le Thuc V, Chantelot C, Chechin D, Cotten A. Diffusion tensor imaging and tractography of the median nerve in carpal tunnel syndrome: preliminary results. Eur Radiol 2008;18:2283-91.

14. Lehmann HC, Zhang J, Mori S, Sheikh KA. Diffusion tensor imaging to assess axonal regeneration in peripheral nerves. Exp Neurol 2010;223:238-44.

15. Eguchi Y, Ohtori S, Orita S, et al. Quantitative evaluation and visualization of lumbar foraminal nerve root entrapment by using diffusion tensor imaging: preliminary results. AJNR Am J Neuroradiol 2011;32:1824-9.

16. Eguchi Y, Oikawa Y, Suzuki M, et al. Diffusion tensor imaging of radiculopathy in patients with lumbar disc herniation: preliminary results. Bone Joint J 2016;98-B:387-94.

17. Balbi V, Budzik JF, Duhamel A, Bera-Louville A, Le Thuc V, Cotten A. Tractography of lumbar nerve roots: initial results. Eur Radiol 2011;21:1153-9.

18. Van der Jagt PK, Dik P, Froeling M, et al. Architectural configuration and microstructural properties of the sacral plexus: a diffusion tensor MRI and fiber tractography study. Neuroimage 2012;62:1792-9.
19. Budzik JF, Verclytte S, Lefebvre G, Monnet A, Forzy G, Cotten A. Assessment of reduced field of view in diffusion tensor imaging of the lumbar nerve roots at 3 T. Eur Radiol 2013;23:1361-6.

20. Dallaudière B, Lincot J, Hess A, et al. Clinical relevance of diffusion tensor imaging parameters in lumbar disco-radicular conflict. Diagn Interv Imaging 2014;95:63-8.

21. Kanamoto H, Eguchi Y, Oikawa Y, et al. Visualization of lumbar nerves using reduced field of view diffusion tensor imaging in healthy volunteers and patients with degenerative lumbar disorders. Br J Radiol 2017;90:20160929.

22. Moses RA, Zhao W, Staub LP, Melloh M, Barz T, Lurie JD. Is the sedimentation sign associated with spinal stenosis surgical treatment effect in SPORT? Spine (Phila Pa 1976) 2015;40:129-36.

23. Hamanishi C, Matukura N, Fujita M, Tomihara M, Tanaka S. Cross-sectional area of the stenotic lumbar dural tube measured from the transverse views of magnetic resonance imaging. J Spinal Disord 1994;7:388-93.

24. Inui Y, Doita M, Ouchi K, Tsukuda M, Fujita N, Kurosaka $\mathrm{M}$. Clinical and radiologic features of lumbar spinal stenosis and disc herniation with neuropathic bladder. Spine (Phila Pa 1976) 2004;29:869-73. 